\title{
First-Principles Study on Migration of Vacancy in Tungsten ${ }^{*)}$
}

\author{
Yasuhiro ODA $^{1)}$, Atsushi M. ITO ${ }^{1)}$, Arimichi TAKAYAMA ${ }^{1)}$ and Hiroaki NAKAMURA ${ }^{1,2)}$ \\ 1) National Institute for Fusion Science, 322-6 Oroshi-cho, Toki 509-5292, Japan \\ ${ }^{2)}$ Nagoya University, 322-6 Oroshi-cho, Toki 509-5292, Japan
}

(Received 10 December 2013 / Accepted 28 April 2014)

\begin{abstract}
We calculated the binding and migration energies of mono-vacancies and di-vacancies in tungsten material using density functional theory. Mono-vacancies diffuse in the [111] direction easier than in the [001] direction. The migration energies of mono-vacancies and di-vacancies are almost the same; moreover, the migration of mono-vacancies and di-vacancies is nearly similar. The di-vacancy binding energies are almost zero or negative. The interactions between two vacancies in tungsten material are repulsive from the second to the fifth nearest neighbors. The vacancies are difficult to aggregate because di-vacancies are less stable than mono-vacancies.
\end{abstract}

(c) 2014 The Japan Society of Plasma Science and Nuclear Fusion Research

Keywords: tungsten, vacancy, helium bubble, density functional theory, nudged elastic band

DOI: 10.1585/pfr.9.3401117

\section{Introduction}

In ITER, pure tungsten and tungsten alloys are promising candidates for divertor plates. When tungsten materials are exposed to high temperatures, thermal vacancies can be observed. The diffusion and aggregation of vacancies cause embrittlement. Moreover, the diffusion of vacancies in tungsten materials is effective against the formation of helium bubbles [1] and tungsten fuzzy nanostructures [2]. Fuzzy nano-structures cause embrittlement in the divertor materials, which subsequently embrittlement causes the release of impurities in the vessel. However, from the viewpoint of nano-device applications, the fuzzy structures can be used as nano-catalysts and stray light measuring devices because tungsten materials with fuzzy structures have large surface area and nearly zero reflectance. Clearly, it is necessary to understand the formation mechanism of nanostructures in tungsten. The key point in bubble formation is the trapping of helium atoms in thermal vacancies in the tungsten lattice structure. It has been pointed out that the helium bubbles influence the formation of fuzzy structures.

The calculations of Takayama et al. [3] by using the density functional theory (DFT) showed that helium atoms aggregate in mono-vacancies. Moreover, the DFT calculations performed by Becquart et al. [4] and Tamura et al. [5] implied that helium atoms also aggregate in interstitial sites; i.e., helium atoms self-aggregate without vacancies. Therefore, to understand the formation of helium bubbles, we have to understand whether the self-aggregation of helium atoms or the aggregation of the vacancies is dominant.

The aggregation of the helium atoms and vacancies can be evaluated from the time scales of movement and

author'se-mail: teamMD@nifs.ac.jp

${ }^{*}$ This article is based on the presentation at the 23rd International Toki Conference (ITC23). binding. The time scales of movement are controlled by the energy of migration of energy. If the migration energy is small, the helium atoms and vacancies aggregate fast. The time scale for maintaining bound state of clusters depends on the binding energy. If the binding energy is large, the clusters of helium atoms are maintained for a long time. Thus, the migration and binding energies need to be studied.

In this study, we have evaluated the migration energies of the vacancies and di-vacancies and the binding energy of di-vacancies in tungsten materials using first-principles calculations based on DFT [6,7], which is a powerful tool to estimate the energies of materials in an atomic scale. For example, the vacancy formation energy in tungsten materials [8] was calculated using DFT calculations. The migration energy and reaction path were estimated by combining DFT and the nudged elastic band (NEB) method [9].

\section{Numerical Methods}

Our first-principles total-energy calculations were based on DFT [6,7] using the Open source package for Material eXplorer (OpenMX) [10]. The generalized gradient approximation (GGA) with the PerdewBurke-Ernzerhof (PBE) functional [11] was used for the exchange-correlation potential. Linear combinations of pseudo-atomic localized orbitals [12], norm-conserving pseudo-potentials [13,14], and projector expansions [15] were used for the core Coulomb potential.

Total energies were calculated for a bcc supercell composed of 128 tungsten atoms $(4 \times 4 \times 4)$. The Monkhorst-Pack method was used for sampling $4 \times 4 \times 4$ $k$-points in the Brillouin zone. The cutoff energy was set at $5918 \mathrm{eV}$. The equilibrium lattice parameter was $3.186 \AA$.

Note that OpenMX uses numerical pseudo-atomic orbitals (PAOs) as the basic function to expand one-particle 
Kohn-Sham wave functions. We employ the radial functions for the $3 s, 2 p, 2 d$, and $1 f$ states with a cut-off radius of $3.71 \AA$ for tungsten. All relaxation procedures continued until the forces on the atoms were less than $5.14 \times 10^{-3} \mathrm{eV} / \AA$.

The binding energies of di-vacancies are given as follows:

$$
E_{b}=2 \times E_{127}-\left(E_{126}+E_{128}\right)
$$

where $E_{128}, E_{126}$, and $E_{127}$ represent the total energy of the supercell without the vacancies, with di-vacancies, and mono-vacancies, respectively.

The vacancy migration energies in tungsten materials were determined using the NEB method [9]. The movement of mono-vacancy at the atomic scale implies that a neighboring atom moves to the vacancy. The movement of di-vacancy in the atomic scale means that a neighbor atom moves on the di-vacancy area. Therefore, the migration energies of the mono-vacancies and di-vacancies are calculated as the migration energies of the atoms adjacent to the vacancies.

\section{Results and Discussion}

The migration of a single vacancy in a bcc lattice has only two possible migration pathways; i.e., along the [001] and [111] directions (Figs. 1 (b) and 1 (c)). Figure 1 (a) shows the total energies of the intermediate states along reaction paths where a mono-vacancy moves to the neighboring site in the [111] and [001] directions. The migration energy of mono-vacancies in the [111] and [001] directions is $1.78 \mathrm{eV}$ and $5.54 \mathrm{eV}$, respectively. The migration energy in the [111] direction is substantially similar to the results of Ahlgren et al. [16]. Thus, a mono-vacancy diffuses in the [111] direction more easily than in the [001] direction.

Figure 2 shows the initial and final paths of the NEB calculations. In the NEB calculations, we need to set the initial path, which has no physical meaning. The initial paths were set by interpolating between structures before and after moving a tungsten atom. The initial paths have been indicated in the left side of Fig. 2 to show that the artificial paths were not selected. By the structure relaxation of the path images bound by the elastic band, the final paths, as shown in the right side of Fig. 2, were obtained.

As shown in Fig. 2, the final paths of the NEB show that the tungsten atom moves in the [111] direction even if the moving distance is long. A tungsten atom moves in the [001] direction only when it cannot move in the [111] direction.

Figure 3 shows the migration energies of the paths shown in Fig. 2. There are nine images for the paths shown in Figs. 2 (a), 2 (b), and 2 (c). However, the numbers of images were increased to 17 to increase the resolution in the calculations of Fig. 3 (a). As shown in Fig. 3, the migration energies of the paths shown in Fig. 2 are almost the same as in the case of mono-vacancy. The migration energies (a)

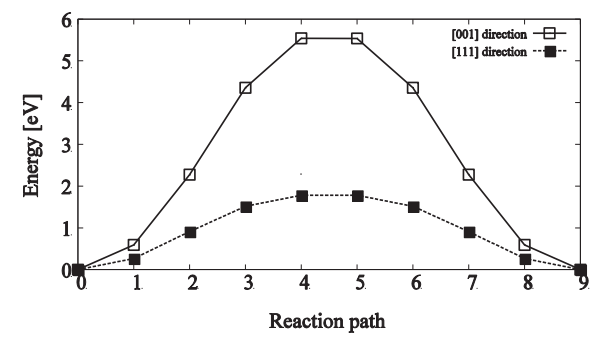

(b)

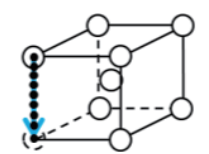

(c)

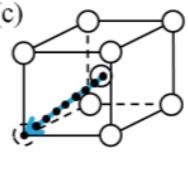

Fig. 1 Migration energies and paths in the NEB calculation: (a) migration energies in the [001] and [111] directions; and (b) and (c) reaction paths for NEB in the [001] and [111] directions, respectively. Migration energies in the [111] and [001] directions are $1.78 \mathrm{eV}$ and $5.54 \mathrm{eV}$, respectively. The mono-vacancy diffuses in the [111] direction easier than in the [001] direction.

(a)

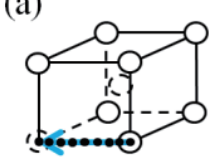

(b)

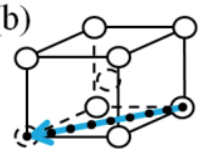

(c)
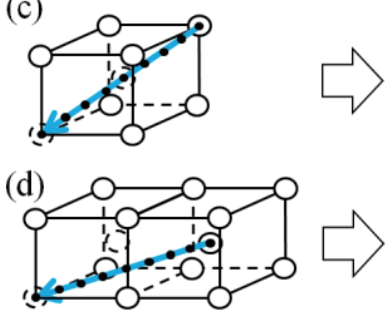
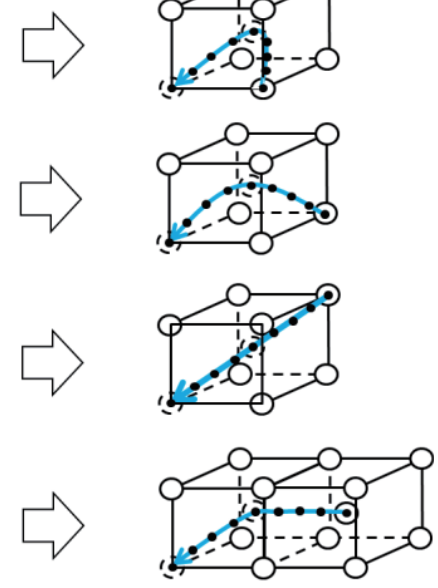

Fig. 2 Migration paths of a di-vacancy in the NEB calculations. The left and right sides are the initial and final paths in the NEB calculations, respectively. Open circles and dashed circles represent tungsten atoms and vacancies, respectively. The dots on the blue line denote the interstitial sites in the NEB calculations.

of paths (a) and (b) are $1.75 \mathrm{eV}$ and $1.73 \mathrm{eV}$, respectively. The migration energy of path (c) is $1.65 \mathrm{eV}$. The vacancies are arranged in a straight line in path (c); thus, the fourth and twelfth images are local minima in the symmetry of the structure.

The migration energy of path (c) is slightly smaller than that of paths (a) and (b). The migration energy shown in Fig. 3(b) is the same as those in the [111] and [001] directions of the mono-vacancy; i.e., the migration of a di-vacancy is approximately the same as that of a monovacancy.

The di-vacancy binding energy for different configurations (see Fig. 4) has been calculated using Eq. (1) and listed in Table 1. The interactions between two vacancies in tungsten materials are surprisingly repulsive from 
(a)

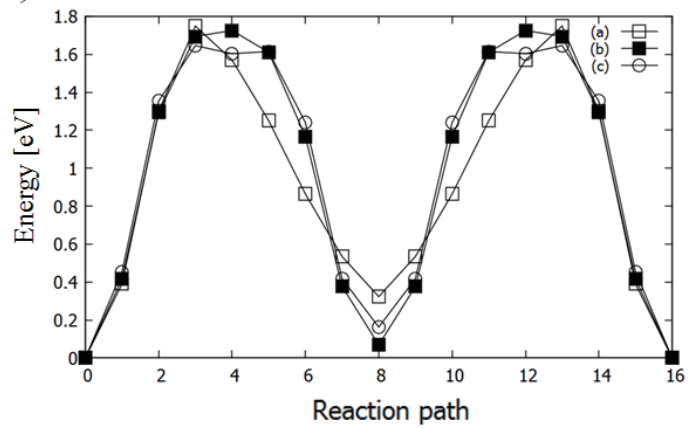

(b)

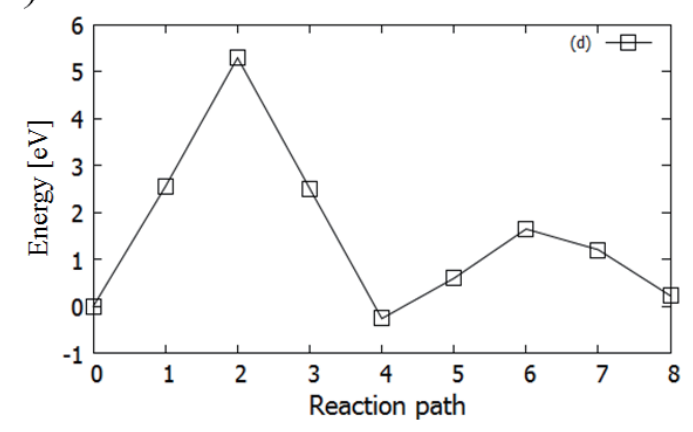

Fig. 3 Migration energies of the paths of Fig. 2. The migration energies are almost the same as that of the mono-vacancy. Graph legends ((a), (b), (c), and (d)) correspond to the legend of Fig. 2.

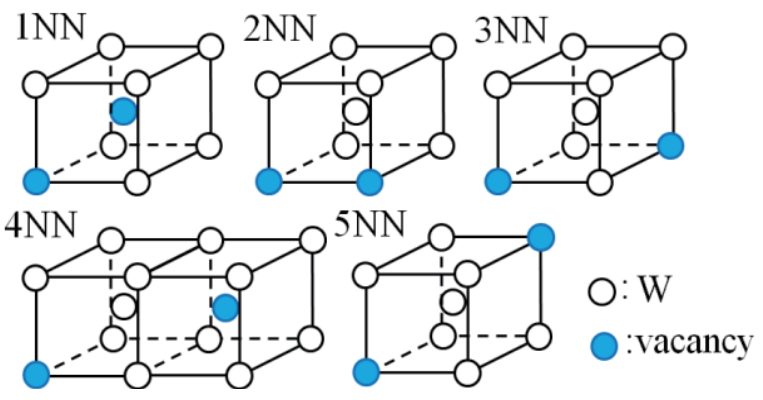

Fig. 4 Di-vacancy configurations from the first nearest neighbor $(1 \mathrm{NN})$ to the fifth nearest neighbor $(5 \mathrm{NN})$ positions. Open circles and blue circles denote tungsten atoms and vacancies, respectively.

Table 1 Binding energies of a di-vacancy. The interactions between two vacancies in tungsten material are repulsive from the second $(2 \mathrm{NN})$ to fifth nearest-neighbor $(5 \mathrm{NN})$. The second nearest-neighbor di-vacancy is the most repulsive.

\begin{tabular}{|l|c|c|c|c|c|}
\hline & $1 \mathrm{NN}$ & $2 \mathrm{NN}$ & $3 \mathrm{NN}$ & $4 \mathrm{NN}$ & $5 \mathrm{NN}$ \\
\hline $\begin{array}{l}\text { Binding } \\
\text { energy } \\
{[\mathrm{eV}]}\end{array}$ & 0.03 & -0.28 & -0.01 & -0.04 & -0.08 \\
\hline
\end{tabular}

the second to the fifth nearest neighbors, where the second nearest neighbor di-vacancy is the most repulsive. Similar to Becquart et al. [17], the di-vacancy binding ener-

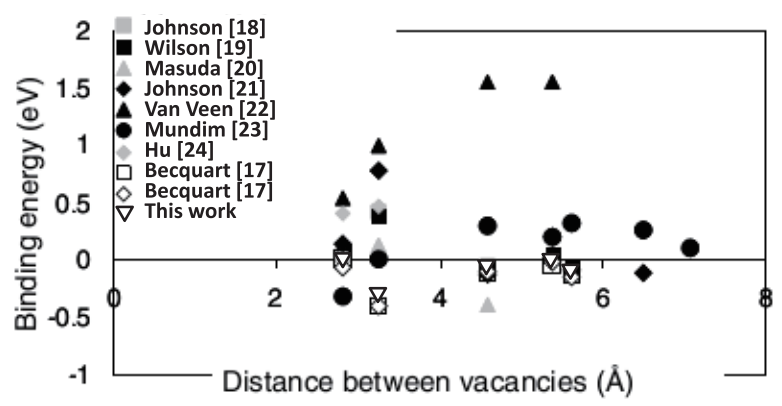

Fig. 5 Binding energies of di-vacancies as a function of the position of the vacancies. We and Becquart et al. [17] calculated the binding energy of a di-vacancy using DFT calculation. Others calculated the binding energy using empirical potentials or semi-empirical calculations [18-24].

gies obtained in this study are almost zero or negative (see Fig. 5). In tungsten materials, a di-vacancy is less stable than a mono-vacancy. Thus, the vacancies in tungsten materials are difficult to aggregate.

We have calculated the binding and migration energies of the vacancies. By comparing the energies between the helium atoms and vacancies, we examined whether the self-aggregation of helium or the aggregation of vacancies is dominant in forming helium bubbles.

The migration energy of the helium atom in tungsten materials calculated by Becquart et al. [4] is $0.06 \mathrm{eV}$, whereas the migration energy of the vacancy calculated in this study is at least $1.8 \mathrm{eV}$. By comparing these values, the time scales of the movement of helium atoms are found to be significantly shorter than those of the vacancies.

By the DFT calculations of Becquart et al. [4] and Tamura et al. [5], the binding energy of helium atoms at interstitial sites is about $1-2 \mathrm{eV}$, whereas the binding energies of vacancies obtained in this work are almost zero or negative. By comparing the binding energies, the time scales for maintaining the bound state of helium clusters are found to be significantly longer than the time scales for maintaining the vacancies.

Consequently, in terms of the time scales of movement and maintaining the bound state, the self-aggregation of helium atoms is easier than the aggregation of vacancies. Thus, we conclude that the self-aggregation of helium atoms is the dominant process in the formation of helium bubbles.

\section{Conclusion}

Point defect calculations were performed using the DFT calculations for tungsten. Mono-vacancies diffuse in the [111] direction easier than in the [001] direction. The migration of mono-vacancies and di-vacancies is approximately the same. In tungsten material, the vacancies are difficult to aggregate because di-vacancies are less stable than mono-vacancies. By comparing the migration and binding energies of the vacancies with those of helium 
atoms, the self-aggregation of helium atoms is found to be more dominant than the aggregation of vacancies in the formation of helium bubbles.

\section{Acknowledgment}

A part of this work was carried out using the Plasma Simulator at the National Institute for Fusion Science and the HELIOS supercomputer system at the Computational Simulation Centre of International Fusion Energy Research Centre (IFERC-CSC), Aomori, Japan, under the Broader Approach collaboration between Euratom and Japan, implemented by Fusion for Energy and JAEA.

[1] S.K. Das and M. Kaminsky, Adv. Chem. Ser. 158, 112 (1976).

[2] S. Kajita, T. Saeki, Y. Hirahata, M. Yajima, N. Ohno, R. Yoshihara and N. Yoshida, Jpn. J. Appl. Phys. 50, 08JG01 (2011).

[3] A. Takayama, A.M. Ito, S. Saito, N. Ohno and H. Nakamura, Jpn. J. Appl. Phys. 52, 01 AL03 (2013).

[4] C.S. Becquart and C. Domain, Phys. Rev. Lett. 97, 97196402 (2006).

[5] T. Tamura, R. Kobayashi, S. Ogata and A.M. Ito, Model. Simul. Mater. Sci. Eng. 22, 015002 (2014).

[6] P. Hohenberg and W. Kohn, Phys. Rev. B 136, 864 (1964).

[7] W. Kohn and L.J. Sham, Phys. Rev. A 140, 1133 (1965).
[8] D. Kato, H. Iwakiri and K. Morishita, J. Nucl. Mater. 417, 1115 (2011).

[9] G. Henkelman and H. Jonsson, J. Chem. Phys. 113, 9978 (2000).

[10] OpenMX web-site:http://www.openmx-square.org/

[11] J.P. Perdew, K. Burke and M. Ernzerhof, Phys. Rev. Lett. 77, 3865 (1996).

[12] T. Ozaki, Phys. Rev. B 67, 155108 (2003).

[13] T. Ozaki and H. Kino, Phys. Rev. B 69, 195113 (2004).

[14] I. Morrison, D.M. Bylander and L. Kleinman, Phys. Rev. B 47, 6728 (1993).

[15] T. Ozaki and H. Kino, Phys. Rev. B 72, 045121 (2005).

[16] T. Ahlgren, K. Heinola, N. Juslin and A. Kuronen, J. Appl. Phys. 107, 033516 (2010).

[17] C.S. Becquart and C. Domain, Nucl. Instrum. Methods Phys. Res. B 255, 23 (2007).

[18] R.A. Johnson and W.D. Wilson, in: P.C. Gehlen, J.R. Beeler, R.I. Jaffee (Eds.), Interatomic Potentials and Simulation of Lattice Defects (Plenum, New York, 1972) p.301.

[19] W.D. Wilson and C.L. Bisson, Rad. Eff. 22, 63 (1974).

[20] K. Masuda, J. Phys. 43, 921 (1982).

[21] R.A. Johnson, Phys. Rev. B 27, 2014 (1983).

[22] A. van Veen, Mater. Sci. Forum 3, 15 (1987).

[23] K.C. Mundim, L.A. Malbouisson, S. Dorfman, D. Fuks, J. Van Humbeek and V. Liubich, J. Mol. Struct. (Theochem.) 539, 191 (2001).

[24] W. Hu, W. Shu and B. Zhang, Comput. Mater. Sci. 23, 175 (2002). 